Open Access

\title{
Low prevalence of hepatitis $C$ co-infection in recently HIV-infected minority men who have sex with men in Los Angeles: a cross-sectional study
}

Kara W. Chew ${ }^{1 *}$, Martha L. Blum²,3, Marjan Javanbakht ${ }^{4}$, Laurel E. Clare ${ }^{2}$, Lorelei D. Bornfleth², Robert Bolan ${ }^{5}$, Debika Bhattacharya ${ }^{2}$ and Pamina M. Gorbach ${ }^{4,6}$

\begin{abstract}
Background: Geographic and sociodemographic characterization of hepatitis C virus (HCV) transmission amongst men who have sex with men (MSM) has been limited. Our aim was to characterize HCV prevalence, risk factors for HCV co-infection, and patterns of HIV and HCV co-transmission and transmitted drug resistance mutations (DRMs) in newly HIV-diagnosed Los Angeles MSM.

Methods: Viral RNA was extracted from stored plasma samples from a Los Angeles cohort of newly diagnosed HIVinfected MSM with well-characterized substance use and sexual behavioral characteristics via computer-assisted selfinterviewing surveys. Samples were screened for HCV by GPCR. HCV E1, E2, core,

NS3 protease and NS5B polymerase and HIV-1 protease and reverse transcriptase regions were amplified and sequenced. Phylogenetic analysis was used to determine relatedness of HCV and HIV-1 isolates within the cohort and viral sequences were examined for DRMs.

Results: Of 185 newly HIV-diagnosed MSM, the majority (65\%) were of minority race/ethnicity and recently infected (57.8\%), with median age of 28.3 years. A minority (6.6\%) reported injection drug use (IDU), whereas 96 (52.8\%) reported recent substance use, primarily cannabis or stimulant use. High risk sexual behaviors included 132 (74.6\%) with unprotected receptive anal intercourse, 60 (33.3 \%) with group sex, and 10 (5.7 \%) with fisting. Forty-five (24.3\%) had acute gonorrhea or chlamydia infection. Only 3 (1.6 \%) subjects had detectable HCV RNA. Amongst these subjects, HIV and HCV isolates were unrelated by phylogenetic analysis and none possessed clinically relevant NS3 or NS5B HCV DRMs.

Conclusions: Prevalence of HCV co-infection was low and there was no evidence of HIV-HCV co-transmission in this cohort of relatively young, predominantly minority, newly HIV-diagnosed MSM, most with early HIV infection, with high rates of high risk sexual behaviors, STI, and non-IDU. The low HCV prevalence in a group with high-risk behaviors for non-IDU HCV acquisition suggests an opportune time for targeted HCV prevention measures.
\end{abstract}

Keywords: Hepatitis C, HIV, Transmission, Non-injection drug use, Men who have sex with men

\footnotetext{
* Correspondence: kchew@mednet.ucla.edu

Prior Presentation: Conference on Retroviruses and Opportunistic Infections

(CROI), Seattle, WA, February 23-26, 2015

'Department of Medicine, David Geffen School of Medicine at UCLA, 11075

Santa Monica Blvd, Suite 100, Los Angeles, CA 90025, USA

Full list of author information is available at the end of the article
} 


\section{Background}

Hepatitis $\mathrm{C}$ virus (HCV) co-infection is common amongst HIV-infected persons, affecting an estimated 4 to 5 million persons worldwide, and is associated with increased morbidity and mortality [1, 2]. Whereas the primary route of $\mathrm{HCV}$ transmission remains injection drug use (IDU), over recent years there has been increasing evidence of sexual transmission among HIV-infected men who have sex with men (MSM), likely driven by mucosal risk factors, including unprotected and traumatic sexual practices in the context of multiple partners, noninjection drug use, and sexually transmitted infections [3-7]. Prevalence estimates for $\mathrm{HCV}$ co-infection in HIV-infected MSM have ranged from 6 to $15.7 \%$, with limited geographic characterization [8-12]. The prevalence of HCV co-infection in HIV-infected MSM in Los Angeles County (LAC) in the U.S. has not been defined, despite LAC being the second largest epicenter for AIDS cases nationally, with high rates of non-injection drug use and high-risk sexual practices. Our aims were to characterize the prevalence of and risk factors for $\mathrm{HCV}$ co-infection and patterns of HIV and HCV co-transmission and drug resistance mutations (DRMs) in a cohort of newly HIV-infected or HIV-diagnosed Los Angeles MSM.

\section{Methods}

Between February 2009 and May 2012 we enrolled participants from a community-based organization providing sexual health services to the gay community in LAC: The Los Angeles LGBT Center. Clients were eligible if they were recently HIV-infected ( $<1$ year of infection) or newly HIV-diagnosed, at least 18 years of age, male, and reported sex with a male partner in the past year. Recent infection was determined by one of four ways: nucleic acid amplification test with negative serology, detuned assay (Low Sensitivity Vitros ECi, Ortho Clinical Diagnostics, Rochester, NY), provider verification, or negative HIV testing within the past year. Plasma was collected and syphilis, chlamydia, and gonorrhea testing performed for all participants. Behavioral and sociodemographic data were collected via computer-assisted self-interview. Minority race/ethnicity was defined as self-identified African-American race or Hispanic ethnicity.

Viral RNA was extracted from stored plasma using the UltrasSens Viral Isolation kit (Qiagen), converted to cDNA using Superscript III Reverse Transcriptase (Invitrogen), and screened for HCV by quantitative polymerase chain reaction (PCR) designed to detect the HCV 5'UTR. The HCV E1/E2/core, NS3 and NS5B regions from HCV positive samples and HIV-1 protease and RT regions from all samples were amplified using either Phusion HighFidelity DNA Polymerase (New England BioLabs) or KOD High-Fidelity Polymerase (Novagen). PCR products were directly sequenced using the BigDye v3.1 Kit (Applied Biosystems).

Sequences were checked for quality, edited, and alignments performed using Chromas Lite (Technelysium Pty Ltd, South Brisbane, Australia) and BioEdit (Ibis Biosciences, Carlsbad, CA). Consensus sequences of an 857 nucleotide (nt) fragment of the HCV NS3 protease gene spanning amino acids 13-297 were aligned with genotype consensus sequences obtained from the Los Alamos National Laboratory (LANL) HCV database (http://www.hiv. lanl.gov), JFH-1, and additional HCV sequences collected from the Los Angeles area. A $1302 \mathrm{nt}$ fragment of the pol gene (HXB2 reference location nt 2254-3555) was aligned with the Clade B consensus sequences obtained from the LANL HIV database. Neighbor-joining phylogenetic trees were generated using the DNAdist and Neighbor programs of PHYLIP. Sequences were examined for the following HCV DRMs: V36M/A, T54A/S, V55A, Q80K, R155K, A156S/T, D168T/V, I/V170A and S282T. HIV-1 DRMs were determined using the Stanford University HIV Drug Resistance Database (http://hivdb.stanford.edu/).

The study was reviewed and approved by the University of California, Los Angeles Institutional Review Board (IRB\# 10-000806) and the Los Angeles LGBT Center Review Committee. Written informed consent was obtained from each study participant, including permission to use stored plasma samples for research testing.

\section{Results}

One hundred eight-five subjects were included in this study. Sociodemographic, behavioral, and clinical characteristics are summarized in Table 1. Median age (interquartile range, IQR) was 28.3 (24.7-35.0 years) and the majority were of minority race or ethnicity $(66.9 \%)$ and recently HIV-infected (57.8\%). At the time of plasma collection, 24 (13.7 \%) reported being prescribed antiretroviral therapy (ART). Median number of partners within the past 12 months was 9 (IQR 4-20), with unprotected receptive or insertive anal intercourse (URAI or UIAI) reported by $132(74.6 \%)$ and 115 (64.6\%), respectively. Forty-five $(24.3 \%)$ subjects tested positive for gonorrhea or chlamydia, with $47(27.5 \%)$ testing positive for syphilis at baseline. A minority (6.6 \%) reported IDU in the past 12 months, whereas 96 (52.8\%) reported recent substance use, primarily cannabis or stimulant use.

Only 3 (1.6\%) subjects had detectable HCV RNA. HCV viral load ranged from 67,000 to 2.2 million copies $/ \mathrm{ml}$. There were too few HCV infections to identify significant risk factors for $\mathrm{HCV}$ co-infection. Of the $3 \mathrm{HCV}$-positive subjects, all were of minority race or ethnicity; one subject was classified as newly HIV infected by detuned assay and two were newly diagnosed with HIV infection of unknown duration. Only 1 reported a history of IDU. All 3 subjects reported non-injection drug use, including 
Table 1 Baseline characteristics of the cohort, overall and by hepatitis C virus (HCV) status

\begin{tabular}{|c|c|c|c|}
\hline Characteristic & $\begin{array}{l}\text { Total }(N=185) n^{a}(\%) \\
\text { or median (IQR) }\end{array}$ & $\begin{array}{l}\text { HCV negative }(N=182) n^{a}(\%) \\
\text { or median (IQR) }\end{array}$ & $\begin{array}{l}\text { HCV positive }(N=3) n^{a}(\%) \\
\text { or median (IQR) }\end{array}$ \\
\hline \multicolumn{4}{|l|}{ Sociodemographics } \\
\hline Age (years) & $28.3(24.7-35.0)$ & $28.3(24.7-34.8)$ & $35.8(22.3-45.1)$ \\
\hline \multicolumn{4}{|l|}{ Race } \\
\hline African American & $32(17.7)$ & $31(17.4)$ & $1(33.3)$ \\
\hline Hispanic & $89(49.2)$ & $87(48.9)$ & $2(66.7)$ \\
\hline White & $47(26.0)$ & $47(26.4)$ & $0(0)$ \\
\hline Asian & $5(2.8)$ & $5(2.8)$ & $0(0)$ \\
\hline Other & $8(4.4)$ & $8(4.4)$ & $0(0)$ \\
\hline Incarcerated, past 12 months & $17(9.3)$ & $17(9.5)$ & $0(0)$ \\
\hline \multicolumn{4}{|l|}{ Substance Use, past 3 months } \\
\hline Any drugs (including cannabis) & $96(52.8)$ & $93(52.0)$ & $3(100)$ \\
\hline Cannabis & $79(43.4)$ & $77(43.0)$ & $2(66.7)$ \\
\hline Cocaine & $26(14.3)$ & $25(14.0)$ & $1(33.3)$ \\
\hline Methamphetamine & $33(18.1)$ & $30(16.8)$ & $3(100)$ \\
\hline Inhalants & $6(3.3)$ & $5(2.8)$ & $1(33.3)$ \\
\hline Sedatives & $16(8.8)$ & $15(8.4)$ & $1(33.3)$ \\
\hline Hallucinogens & $3(1.7)$ & $2(1.1)$ & $1(33.3)$ \\
\hline Opioids & $6(3.3)$ & $5(2.8)$ & $1(33.3)$ \\
\hline Injection drug use, past 12 months & $12(6.6)$ & $11(6.2)$ & $1(33.3)$ \\
\hline Partner injection drug use, past 12 months & $34(18.6)$ & $32(17.8)$ & $2(66.7)$ \\
\hline Alcohol use, past 3 months & $129(70.9)$ & $127(71.0)$ & $2(66.7)$ \\
\hline \multicolumn{4}{|l|}{ Sexual Behaviors } \\
\hline Sexual partners, past 12 months & $9(4-20)$ & $9(4-20)$ & $6(6-14)$ \\
\hline Unprotected insertive Al, last 6 partners & $115(64.6)$ & $113(64.2)$ & $2(66.7)$ \\
\hline Unprotected receptive Al, last 6 partners & $132(74.6)$ & $130(74.3)$ & $2(66.7)$ \\
\hline Fisted by partner, last 6 partners & $10(5.7)$ & $10(5.8)$ & $0(0)$ \\
\hline Transactional sex & $20(11.0)$ & 19 (10.6) & $1(33.3)$ \\
\hline Group sex & $60(33.3)$ & $58(32.4)$ & $2(66.7)$ \\
\hline \multicolumn{4}{|l|}{ Sexually Transmitted Infections } \\
\hline Chlamydia, baseline testing & $32(18.2)$ & $30(17.3)$ & $2(66.7)$ \\
\hline Gonorrhea, baseline testing & 19 (10.6) & 19 (10.8) & $0(0)$ \\
\hline Syphilis, TPPA positive, baseline testing & $47(27.5)$ & $46(27.2)$ & $1(33.3)$ \\
\hline Chlamydia, self-reported, past 12 months & $69(37.9)$ & $68(38.0)$ & $1(33.3)$ \\
\hline Gonorrhea, self-reported, past 12 months & $65(35.7)$ & $64(35.8)$ & $1(33.3)$ \\
\hline Syphilis, self-reported, past 12 months & $57(31.3)$ & $56(31.3)$ & $1(33.3)$ \\
\hline HSV, self-reported, past 12 months & $11(6.0)$ & $11(6.2)$ & $0(0)$ \\
\hline HIV viral load, copies $/ \mathrm{mL}^{\mathrm{b}}$ & $41,538(5771-150,756)$ & $41,518(6296-144,378)$ & $60,476(<50-155,000)$ \\
\hline CD4 cell count $\left(\text { cells } / \mathrm{mm}^{3}\right)^{c}$ & $553(408-692)$ & $553(408-692)$ & Unavailable \\
\hline
\end{tabular}

IQR interquartile range, $A l$ anal intercourse, TPPA treponema pallidum particle agglutination, $H S V$ herpes simplex virus

${ }^{a}$ Sum of $n$ may not equal total $\mathrm{N}$ because of missing data; ${ }^{\mathrm{b}}$ Available for 171 of 185 subjects; ${ }^{\mathrm{C}}$ Available for 80 subjects

methamphetamines, within the past 3 months. All 3 subjects also reported high-risk sexual behavior, including UIAI, URAI, and group sex.
Amongst the $3 \mathrm{HCV}$-infected subjects (subjects $\mathrm{A}, \mathrm{B}$, and $\mathrm{C}$ ), HIV and HCV sequences were unrelated by phylogenetic analysis (see Fig. 1). Based on HCV NS3 

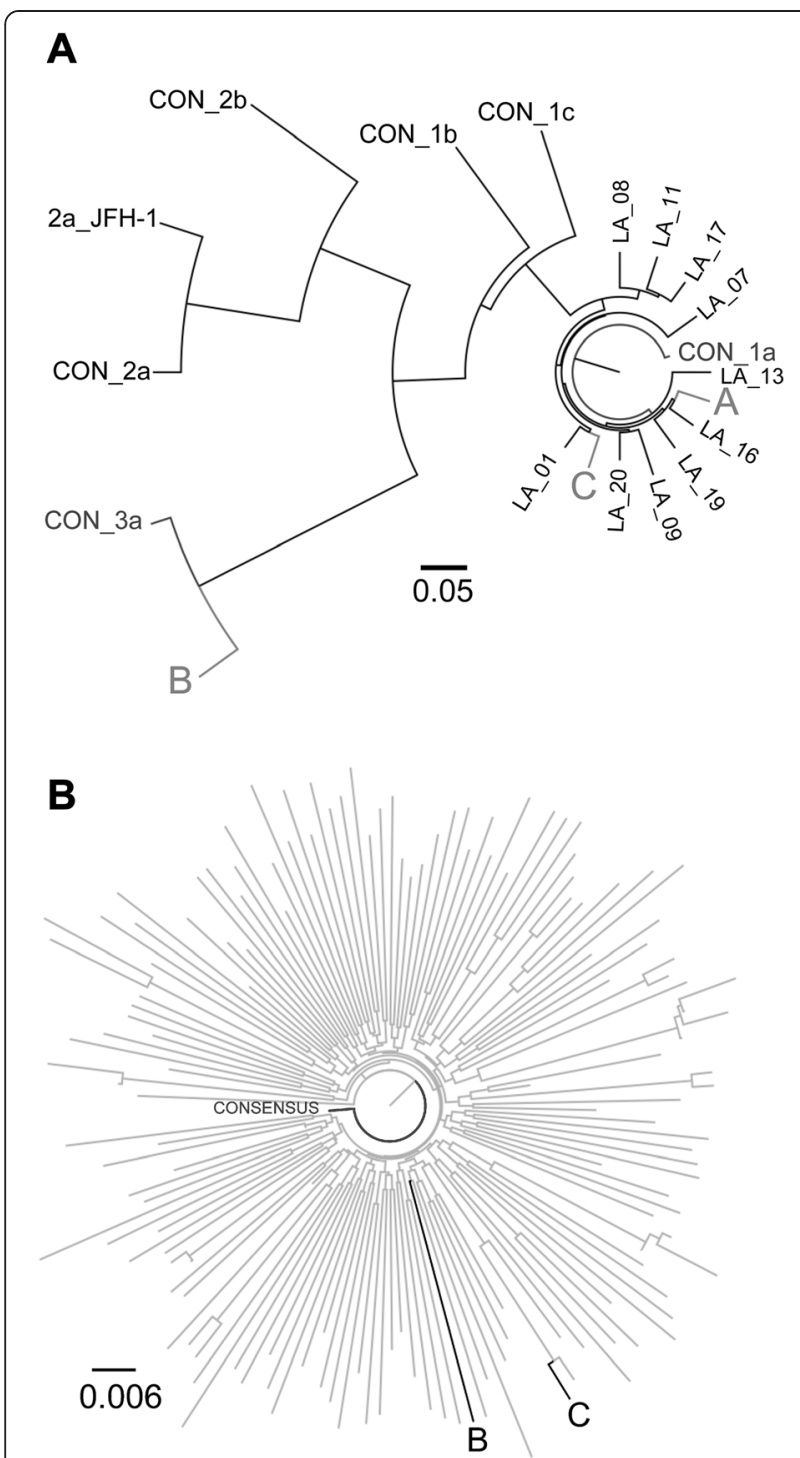

Fig. 1 a Phylogenetic analysis of Hepatitis C virus (HCV) isolates. Consensus sequences of an 857 nucleotide (nt) fragment of the HCV NS3 protease from the three HCV isolates from the cohort were aligned with the Los Alamos National Laboratory HCV Database consensus sequences for HCV genotype (gt) 1 a and $3 \mathrm{a}$, along with additional reference sequences from subjects living in Los Angeles, to make a neighbor-joining tree. The consensus sequences for gt 1a and $3 a$ are labeled as "CON" with the corresponding genotype. The Los Angeles HCV sequences are labeled as "LA" with the corresponding subject number. The HCV-positive subject samples are labeled A, B, and $\mathrm{C}$. The tree is rooted with the HCV genotype 1a consensus sequence and the genetic distance scale bar is located at the center of the figure. $\mathbf{b}$ Phylogenetic analysis of HIV-1 pol. A 1302 nt fragment of pol covering the HIV-1 protease and reverse transcriptase (HXB2 reference location nt 2254-3555) from

$N=148$ isolates from the cohort was aligned with the Clade B consensus and used to build a neighbor-joining tree. Each

sequence is labeled with a unique subject identifier, and the HCVpositive subjects are labeled $A, B$, and $C$. The tree is rooted with the Clade B consensus sequence. The genetic distance scale bar is located at the lower left of the figure protease sequences, a neighbor-joining phylogenetic tree showed that $\mathrm{HCV}$ sequences from subjects $\mathrm{A}$ and $\mathrm{C}$ were most closely related to genotype $1 \mathrm{a}$; and subject $\mathrm{B}$, to genotype $3 \mathrm{a}$. The two genotype 1a-infected subjects had sequences that were no more closely related to each other than to other isolates from the Los Angeles area, indicating that these were not a closely linked transmission pair. Similar results were obtained using a $1784 \mathrm{nt}$ fragment of HCV NS5B and a 2470 nt fragment spanning the E1-E2-Core region of the genome (data not shown). No HIV-1 sequence was obtained from HCVpositive subject $\mathrm{A}$, who was identified at screening as having recently acquired HIV infection, but had initiated antiretroviral therapy with fully suppressed HIV viremia at the time of study enrollment and plasma collection. HIV-1 sequences from subjects $B$ and $C$ were no more closely related to each other than to sequences obtained from other cohort members. However, the HIV-1 sequences from subject $\mathrm{C}$ and another subject (D) were closely related with $100 \%$ bootstrap support and an inter-patient genetic distance of $<1 \%$ strongly indicative of these being a transmission pair. Subject D was HCVnegative, both by qPCR and serologically. One subject, who was ART naïve, had an HIV RT mutation (K103N). None had clinically relevant NS3 or NS5B HCV DRMs.

\section{Discussion}

Prevalence of $\mathrm{HCV}$ co-infection was low and there was no evidence of HIV-HCV co-transmission in this cohort of young, predominantly minority, newly HIV-diagnosed MSM. The majority of subjects had recent HIV infection and notable behavioral and clinical risk factors for sexual $\mathrm{HCV}$ transmission, including high-risk sexual practices, sexually transmitted infections, and non-injection (primarily stimulant) substance use, with low rates of injection drug use. The lower prevalence of HCV compared with other HIV-infected MSM cohorts (1.6 vs 6-15.7\%) [8-12] may reflect the younger age of the cohort with fewer cumulative exposures to $\mathrm{HCV}$, lower rates of IDU, relatively greater immune preservation with earlier HIV infection, and identification of HCV by HCV RNA instead of by serology. In our study, by measuring HCV RNA, we measured prevalence of active $\mathrm{HCV}$ replication and not exposure or infection with possible clearance, as would be measured by serology. Assessment by both serology and HCV RNA would provide broader characterization of $\mathrm{HCV}$ exposure in the cohort, but due to limited sample volume, we could not perform testing for both and elected for HCV RNA testing alone as a measure of active HCV infection and risk for $\mathrm{HCV}$ transmission. Demographically, our cohort differed from others in its geographic and racial/ethnic composition, wherein our cohort was predominantly of minority race and half was Hispanic, as compared to most other reported cohorts that were 
predominantly White. The epidemiology of $\mathrm{HCV}$ coinfection in HIV-infected Hispanic MSM has not been well described. As described by Kunikholm et al., utilizing National Health and Nutrition Examination Survey (NHANES) and Hispanic Community Health Study/Study of Latinos (HCHS/SOL) data, HCV prevalence appears to differ by Hispanic/Latino background and the prevalence of HCV in the West Coast Hispanic population may be lower than in others [13].

While there were too few subjects with HCV infection in the cohort to explore associations between potential behavioral risk factors and $\mathrm{HCV}$ infection, the subjects that did have $\mathrm{HCV}$ co-infection all reported methamphetamine and other non-injection drug use, as well as high-risk sexual practices, consistent with risk factors identified in larger cohorts. The lack of HCV clustering or transmission pairs is likely due to the smaller sample size than in other studies [6,7], as well as may relate uniquely to the geographically wide sexual networks in LAC compared to other urban areas. The lack of evidence of HIV-HCV co-transmission may reflect the low prevalence of $\mathrm{HCV}$ infection in the cohort, but may also suggest that co-transmission occurs infrequently, and that $\mathrm{HCV}$ infection is more often acquired following HIV infection, instead of simultaneously or preceding HIV infection, particularly in non-IDU settings. The lack of HCV DRMs is likely due to the few observed $\mathrm{HCV}$ infections.

\section{Conclusions}

We found a lower than expected prevalence of $\mathrm{HCV}$ infection in our LAC HIV-infected cohort of MSM. By behavioral risk factors, the population studied is at high risk for incident $\mathrm{HCV}$ infection. This presents an opportunity, namely at the time of a new HIV diagnosis and during early HIV infection, for targeted HCV prevention strategies to reduce non-IDU HCV transmission among HIV-infected MSM.

\section{Availability of data and materials}

We are complying with the NIH policy on shared data. HIV and HCV sequences are available in the NIH genetic sequence database GenBank, accession numbers KJ680920 - KJ680970 and KM874782 - KM874789.

\section{Competing interests}

KWC, MLB, MJ, LEC, LDB, RB and PMG have no conflicts of interest to declare. $\mathrm{DB}$ has received research support from Abbvie and Bristol Myers Squibb.

\section{Authors' contributions}

KWC contributed to the design of the study and oversaw data acquisition, data analysis and interpretation, and drafted the manuscript. MLB co-led conception and design of the study, oversaw acquisition of laboratory data, conducted the phylogenetic analyses, contributed to interpretation of the data, and helped draft the manuscript. MJ contributed to the design of the study, conducted data analyses, and helped draft the manuscript. LEC conducted the laboratory assays including viral RNA extraction, CDNA synthesis, PCR, and sequencing, and helped draft the manuscript. LDB helped coordinate and direct the laboratory work, including viral RNA extraction, cDNA synthesis, PCR, sequencing and analysis, and helped draft the manuscript. RB contributed to acquisition and interpretation of the data. DB co-led conception of the study and contributed to the study design and interpretation of the data. PMG is the Principal Investigator of and designed the parent study, including the data collection instruments, provided guidance in the conception, study design, analysis approach, and interpretation of the data, and provided critical revisions for the manuscript. All authors read and approved the final manuscript.

\section{Acknowledgements}

This work was supported by the National Institute of Allergy and Infectious Diseases at the National Institutes of Health (P30 Al028697, Center for AIDS Research supplement to KWC) and the National Institute on Drug Abuse at the National Institutes of Health (R01 DA022116 to PMG).

\section{Author details}

'Department of Medicine, David Geffen School of Medicine at UCLA, 11075 Santa Monica Blvd, Suite 100, Los Angeles, CA 90025, USA. ${ }^{2}$ Department of Medicine, David Geffen School of Medicine at UCLA, 10833 Le Conte Ave, 37-121 CHS, Los Angeles, CA 90095, USA. ${ }^{3}$ Present Address: Community Hospital of the Monterey Peninsula, Infectious Diseases Division, 23625 Holman Highway, Monterey, CA 93942, USA. ${ }^{4}$ Department of Epidemiology, UCLA Fielding School of Public Health, 71-235E CHS, Los Angeles, CA 90095, USA. ${ }^{5}$ Los Angeles LGBT Center, 1625 N. Schrader Blvd, Los Angeles, CA 90028, USA. ${ }^{6}$ Department of Medicine, David Geffen School of Medicine at UCLA, 41-295 CHS, Los Angeles, CA 90095, USA.

Received: 20 July 2015 Accepted: 12 November 2015

Published online: 20 November 2015

\section{References}

1. Berenguer J, Alejos B, Hernando V, Viciana P, Salavert M, Santos I, et al. Trends in mortality according to hepatitis $C$ virus serostatus in the era of combination antiretroviral therapy. AIDS. 2012;26:2241-6.

2. van de Laar TJ, Matthews GV, Prins M, Danta M. Acute hepatitis C in HIV-infected men who have sex with men: an emerging sexually transmitted infection. AIDS. 2010;24(12):1799-812.

3. Yaphe S, Bozinoff N, Kyle R, Shivkumar S, Pai NP, Klein M. Incidence of acute hepatitis $C$ virus infection among men who have sex with men with and without HIV infection: a systematic review. Sex Transm Infect. 2012;88(7):558-64.

4. Rauch A, Rickenbach M, Weber R, Hirschel B, Tarr PE, Bucher HC, et al. Unsafe sex and increased incidence of hepatitis $C$ virus infection among HIV-infected men who have sex with men: the Swiss HIV Cohort Study. Clin Infect Dis. 2005;41(3):395-402.

5. Matthews GV, Pham ST, Hellard M, Grebely J, Zhang L, Oon A, et al. Patterns and characteristics of hepatitis $C$ transmission clusters among HIV-positive and HIV-negative individuals in the Australian trial in acute hepatitis C. Clin Infect Dis. 2011;52(6):803-11.

6. Centers for Disease Control and Prevention (CDC). Sexual transmission of hepatitis C virus among HIV-infected men who have sex with men-New York City, 2005-2010. MMWR Morb Mortal Wkly Rep. 2011;60:945-50.

7. Garg S, Taylor LE, Grasso C, Mayer KH. Prevalent and incident hepatitis C virus infection among HIV-infected men who have sex with men engaged in primary care in a Boston community health center. Clin Infect Dis. 2013;56(10):1480-7.

8. Wandeler G, Gsponer T, Bregenzer A, Gunthard HF, Clerc O, Calmy A, et al, Hepatitis C virus infections in the Swiss HIV Cohort Study: a rapidly evolving epidemic. Clin Infect Dis. 2012;55(10):1408-16.

9. Raymond HF, Chu P, Nieves-Rivera I, Louie B, McFarland W, Pandori M. Hepatitis C infection among men who have sex with men, San Francisco, 2011. Sex Transm Dis. 2012;39(12):985-6.

10. Matser A, Vanhommerig J, van der Loeff MF S, Geskus RB, de Vries HJ, Prins $J M$, et al. HIV-infected men who have sex with men who identify themselves as belonging to subcultures are at increased risk for hepatitis $C$ infection. PLoS One. 2013;8(3):e57740.

11. Urbanus AT, van de Laar TJ, Stolte IG, Schinkel J, Heijman T, Coutinho RA, et al. Hepatitis C virus infections among HIV-infected men who have sex with men: an expanding epidemic. AIDS. 2009;23(12):F1-7. 
12. Kuniholm MH, Jung M, Everhart JE, Cotler S, Heiss G, McQuillan G, et al. Prevalence of hepatitis $C$ virus infection in US Hispanic/Latino adults: results from the NHANES 2007-2010 and HCHS/SOL studies. J Infect Dis. 2014; 209(10):1585-90

13. Franco S, Tural C, Nevot M, Molto J, Rockstroh JK, Clotet B, et al. Detection of a sexually transmitted hepatitis $C$ virus protease inhibitor-resistance variant in a human immunodeficiency virus-infected homosexual man. Gastroenterology. 2014;147(3):599-601. e1.

Submit your next manuscript to BioMed Central and take full advantage of:

- Convenient online submission

- Thorough peer review

- No space constraints or color figure charges

- Immediate publication on acceptance

- Inclusion in PubMed, CAS, Scopus and Google Scholar

- Research which is freely available for redistribution 